SYSTEMATIC REVIEW

\title{
EFFECT OF RESISTANCE TRAINING ON RESPIRATORY PARAMETERS - A SYSTEMATIC REVIEW
}

\begin{abstract}
BACKGROUND AND OBJECTIVES

Normally, aerobic exercises are considered as the therapeutic medium for improving cardiopulmonary parameters. The objective of this review is to evaluate the impact of aerobic resistance training on pulmonary parameters.
\end{abstract}

\section{DATA SOURCES}

Pubmed, Google Scholar, Science Direct, Biomed-Central (BMC), Pubmed-Europe, Medline, EMBASE and Pedro

\section{ELIGIBILITY CRITERIA}

Studies investigating role of resistive exercise training on pulmonary parameters including FVC, FEVI, $\mathrm{VO}_{2}, \mathrm{TLCO}$ etc, either alone or in combination with other exercise regimes, are included.

\section{REVIEW METHODS}

Data was extracted according to a standardized assessment form according to inclusion criteria. Risk of bias was evaluated according to the Cochrane risk of bias guidelines and qualitative analysis was performed.

\section{RESULTS}

Out of 13 selected studies, 4 studies supported the view that resistive exercises have significant role in improving the pulmonary parameters. There was a low risk of bias found among the studies that were selected and included in the review.

\section{CONCLUSION}

The review concludes that there is a low evidence for resistive exercises in improving the pulmonary parameters. However, more researches are needed to be conducted on the subject in future.

\section{KEYWORDS}

Resistance Exercise, Resistive training, FEV1, FVC, spirometric values, respiration, pulmonary parameters, cardiopulmonary endurance

\author{
Saima Ali \\ Physiotherapist \\ Aga Khan University Hospital \\ saimaali_83@hotmail.com \\ Muhammad Ather Hashmi \\ Lecturer \\ Ziauddin College of Rehabilitation Sciences \\ Ziauddin University \\ ather_hashmi@hotmail.com \\ Jaza Rizvi \\ Lecturer \\ Ziauddin College of Rehabilitation Sciences \\ Ziauddin University \\ jaza.rizvi@zu.edu.pk
}

[Ali S, Hashmi MA, Rizvi J. Effect Of Resistance Training On Respiratory Parameters - A Systematic Review. Pak. j. rehabil. 2018;7(1):4-14] 


\section{INTRODUCTION}

Aerobic and anaerobic physical activity is evident for having curative as well as preventive effects on cardiovascular and respiratory systems by enhancing respiratory parameters, aerobic strength and oxygen consumption ${ }^{1-3}$. Predominantly, aerobic exercise are opted for cardiopulmonary endurance consisting of long duration and short intensity, as recommended by American College of Sports Medicine (ACSM) ${ }^{4,5}$. Factually, the positive effects of aerobic exercise in intensifying respiratory strength, pulmonary ventilation, oxygen consumption, enhancing lungs volumes and capillary diameter; and more specifically, its role in improving respiratory indices like Force Vital Capacity (FVC), Force expiratory volume in one Second (FEV1) and maximal voluntary ventilation (MVV); have been establish by the evidence of existing literature ${ }^{6-10}$.

Anaerobic or resistance exercise, due to have focus on muscular strength and endurance too, are becoming an essential component of pulmonary rehabilitation program ${ }^{11,12}$. However, sports like basketball, water polo and rowers have been suggested to be significant in improving functions of pulmonary system and athletes involved in these sports have demonstrated statistically improved vital capacity (VC), force vital capacity (FVC) and force expiratory volumes in one minute $\left(\mathrm{FEV}_{1}\right)$, comparing to those of sedentary but healthy individuals $^{13}$. Despite of all these evidences suggest that strengthening of small and fat free muscles is proved to be beneficial for controlling dyspnea and enhancing functional capacity in patient with COPD $^{14}$

Resistance training of respiratory muscles is thought to be beneficial in improving strength as well as pulmonary parameters ${ }^{15}$. Moreover, one multidisciplinary review identifies musculature of pelvic floor as an important contributor in respiration, which plays a crucial role in generating intra-abdominal pressure ${ }^{16}$

Although, one study strongly recommend the potential effect of resistance training on FEV 1 even if it is not consider to be the direct significance of resistance training but probably due to the improvement in lung physiology and functions as a consequence of pulmonary rehabilitation. Resistance training is considered to be an important treatment intervention in patient with COPD as it has a strong effect in boosting muscular strength and ultimately optimizing functional status ${ }^{17}$.

Moreover, it has been suggested in a research that effect of resistance training in lower extremity is more influential in increasing minute ventilation (VE), O2 uptake, and decreasing dyspnea as compared to aerobic exercise of upper and lower extremities ${ }^{18}$.
Interestingly, a research conducted among the non-active healthy males in Iran evaluated the relationship of resistance training as well as high intensity interval training exercise regimes with respiratory indices and inflammatory markers and concluded that the FEV I, FVC and other spirometric ratios significantly increased in the experimental groups comparing to the control group that receive no exercise ${ }^{19}$.

Furthermore, a study done in 2017 on heart failure patients that significantly proved the fact that effect of combined aerobic and resistance training plus non-invasive ventilation is more influential in controlling dyspnea and quality of life than combined aerobic and resistance training, 6 minute walk test, FEV1 and FVC showed no improvement when compared between these two groups ${ }^{20}$.

Beside these, other regimes of exercise are also conducted to check their effects on respiratory parameters. Therefore, a research was conducted to find out the relationship between the pilates exercise and respiratory parameters in obese sedentary women ${ }^{21}$.

The current systematic review is aimed to derive a qualitative analysis of the recent available studies inquiring whether resistive exercises improve the respiratory parameters or have no effects on them.

\section{METHODOLOGY}

\section{Eligibility criteria and Information Sources}

The systemic review was conducted in accordance with the guidelines provided by PRISMA (Preferred Reporting Items for Systemic Review and Meta-Analysis). An electronic search with related keywords was conducted on various databases including Cochrane, Pubmed, Google Scholar, Science Direct, Biomed-Central (BMC), Pubmed-Europe, Medline, EMBASE and PEdro.

\section{Search of Articles}

Strategies acquired for literature searching include MeSH-terms and keywords using Boolean logic such as Pulmonary Function Test, Respiratory Parameters AND Resistance training, Respiratory Muscle Training, EXERCISE THER. The literature search was customized for the articles published in the duration between 2013 -2018 without any specification of language. However, all full texted articles accessed, were either in English originally or English translated versions were included in the review.

\section{Study Selection}

Apparently, 46 articles were retrieved in all out of which 13 articles were selected according to the inclusion criteria. The researches investigating the effects of resistance training on respiratory parame- 
ters such as Forced Vital Capacity (FVC), Forced Expiration volume in first second (FEV1), Vital Capacity (VC), Minute ventilation (MV), Maximal Voluntary Ventilation (MVV), Maximum Oxygen consumption (VO2max), Maximum Pulmonary Inspiration (PImax), Maximum Pulmonary Expiration (PEmax) and Transfer factor for carbon monoxide (TLCO), among the healthy as well as in diseased individuals, were included without gender segregation.
Study Charactersitics

The characteristics of the researches, included in the review, include 2 quasi-experimental studies while the remaining 11 were Randomized controlled trials (RCTs). 9 of these studies independently designated an experimental group for resistive exercises as intervention while remaining 4 reported to have applied resistive exercise training in combination with other exercise regimes as demonstrated in figure 1.

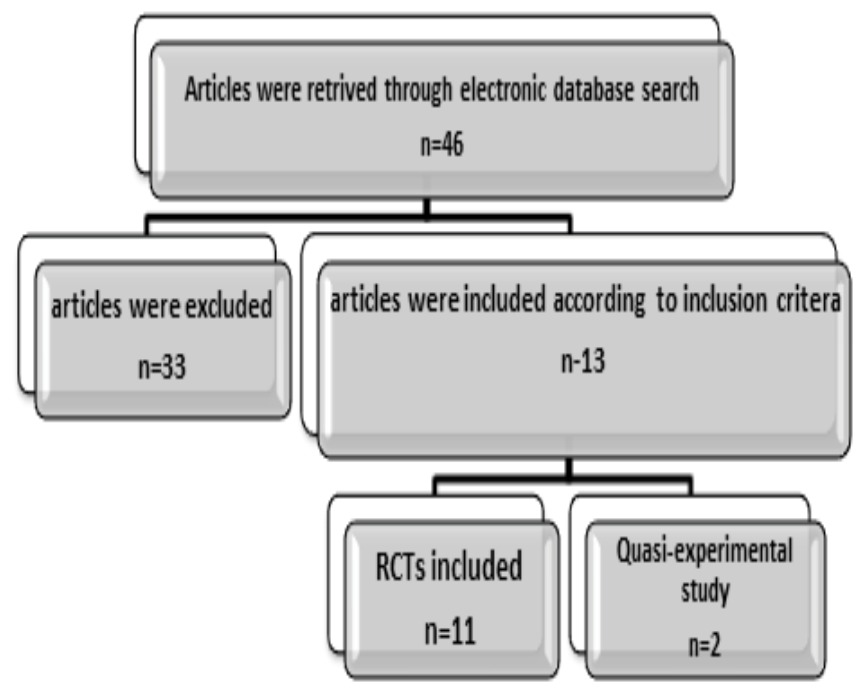

Figure: 1 Flow diagram representing study selection procedure and characteristics

\begin{tabular}{|c|c|c|c|c|c|}
\hline S.no & Author & $\begin{array}{c}\text { Year } \\
\text { of } \\
\text { Pub }\end{array}$ & Group & $n$ & Intervention \\
\hline 1 & Ganesh BR $^{22}$ & 2018 & Experimental & 43 & $\begin{array}{c}10 \text { Lbs. sandbag breathing } \\
\text { exercise for } 5-10 \text { minutes, thrice } \\
\text { a week for } 4 \text { weeks }\end{array}$ \\
\hline \multirow[t]{3}{*}{2} & Molassiotis $A^{23}$ & 2015 & intevention & 18 & $\begin{array}{l}\text { IMT sessions weeky for } 12 \\
\text { weeks for a total of } 30 \text { mins/day }\end{array}$ \\
\hline & & & control & 18 & \\
\hline & Edvardsen $\mathrm{E}^{24}$ & 2014 & training & 30 & $\begin{array}{l}\text { high-intensity endurance and } \\
\text { strength training ( } 60 \text { min, three } \\
\text { times a week, } 20 \text { weeks) }\end{array}$ \\
\hline 4 & Pothirat $C^{25}$ & 2015 & experimental & 30 & $\begin{array}{c}\text { incremental strength and } \\
\text { endurance with two } 35-40- \\
\text { minute sessions per week for } 8 \\
\text { weeks }\end{array}$ \\
\hline \multirow[t]{2}{*}{5} & Santana-Sosa $E^{26}$ & 2013 & training & 10 & $\begin{array}{l}\text { Aerobic + strengthening } \\
\text { Exercise, } 3 \text { day/week }\end{array}$ \\
\hline & & & control & 10 & No intervention \\
\hline 6 & $\begin{array}{l}\text { Zambom- } \\
\text { Ferraresi F }\end{array}$ & 2015 & combined training & 14 & $\begin{array}{l}12 \text { weeks, I d / week resistance } \\
\text { \&1d/ weekendurance training }\end{array}$ \\
\hline
\end{tabular}




\begin{tabular}{|c|c|c|c|c|c|}
\hline & & & resistance training & 14 & $\begin{array}{c}12 \text { weeks, } 2 \mathrm{~d} / \text { week resistance } \\
\text { training }\end{array}$ \\
\hline & & & control & 8 & No intervention \\
\hline \multirow[t]{2}{*}{7} & Rovedder $\mathrm{PME}^{28}$ & 2014 & exercise & 19 & $\begin{array}{c}\text { home based exercise; a } \\
\text { combination of aerobic and } \\
\text { strength training }\end{array}$ \\
\hline & & & control & 22 & normal routine practices \\
\hline \multirow[t]{3}{*}{8} & Moradians $\mathrm{V}^{23}$ & 2016 & aerobic & 12 & $\begin{array}{c}\text { aerobic ex 40-60 } \mathrm{min} 3 / \text { week } \\
(10 \mathrm{~min} \text { WU, } 20 \mathrm{~min} A A @ 70- \\
80 \% \mathrm{MHR}\end{array}$ \\
\hline & & & resistance & 12 & $\begin{array}{l}\text { weight training } 3 \text { sets } 10 \text { reps of } \\
\text { each ex, intensity increased by }\end{array}$ \\
\hline & & & interval & 12 & $\begin{array}{l}\text { Short periods of running at } 85 \% \\
\text { maximum heart rate. Each } \\
\text { session started with warm up, } \\
\text { which was followed by five } \\
\text { rounds of running } 20,30,60 \text {, } \\
\text { and } 100 \text { meters, and finished } \\
\text { with cool down exercises. }\end{array}$ \\
\hline \multirow[t]{2}{*}{9} & Chun SP30 & 2015 & experimental & 15 & $\begin{array}{c}\text { Core stability exercises } 30 \mathrm{~min} / \\
\text { session, } 4 \text { session / week for } 8 \\
\text { weeks }\end{array}$ \\
\hline & & & control & 15 & general exercises \\
\hline \multirow[t]{3}{*}{10} & Vonbank $k^{31}$ & 2012 & $\begin{array}{l}\text { endurance } \\
\text { training }\end{array}$ & 12 & $\begin{array}{c}\text { cycle ergometer twice / week } \\
\text { total duration } 60 \text { min intensity } \\
60 \% \\
\end{array}$ \\
\hline & & & $\begin{array}{c}\text { progressive } \\
\text { strength tralning }\end{array}$ & 12 & twice/week \&-15 rep / set \\
\hline & & & Combination & 12 & both combined \\
\hline \multirow[t]{3}{*}{11} & Tartibian $^{32}$ & 2018 & HIIT & 12 & $\begin{array}{c}n=12,30 \mathrm{~min} / \text { day, } 3 \text { days } / \text { week } \\
\text { at } 60-90 \% \text { of heart rate reserve } \\
\text { for } 8 \text { weeks }\end{array}$ \\
\hline & & & RT & 12 & $\begin{array}{c}\mathrm{n}=12,60 \mathrm{~min} / \text { day, } 3 \text { days } / \text { week } \\
\text { by } 60 \%-90 \% \text { of } 1 \text { MM for } 8 \\
\text { weeks }\end{array}$ \\
\hline & & & NON-EX & 12 & No Interventlon \\
\hline \multirow[t]{3}{*}{12} & Maryam ${ }^{33}$ & 2013 & Control & 9 & no intervention \\
\hline & & & ET & 9 & $\begin{array}{l}\text { (8 weeks, } 3 \text { sessions/week) for } \\
\text { ET was } 20-26 \mathrm{~min} / \mathrm{session} \\
\text { running with } 60-80 \% \text { maximum } \\
\text { heart rate ( } \mathrm{HR} \text { max }\end{array}$ \\
\hline & & & RT & 10 & $\begin{array}{l}\text { two circuits/session, } 40-60 \text { s for } \\
\text { each exercise with } 60-80 \% \text { one } \\
\text { repetition maximum (1RM), } 1 \\
\text { and } 3 \text { minutes active rest } \\
\text { between exerclses and clrcults } \\
\text { respectively }\end{array}$ \\
\hline
\end{tabular}




\begin{tabular}{|c|c|c|c|c|c|}
\hline & & ERT & 9 & $\begin{array}{c}\text { in agreement with either ET or } \\
\text { RT protocols, but the times of } \\
\text { running and circuits were half of } \\
\text { ET and RT }\end{array}$ \\
\hline 13 & Aweto HA & 2015 & A: aerobic & 15 & $\begin{array}{c}\text { Aerobic exercise; treadmill, 10 } \\
\text { min for 4week, 15min for 2 } \\
\text { week, twice a for 6 week total }\end{array}$ \\
\hline & & & B: resistance & 15 & $\begin{array}{c}\text { Dumbells, 50-70\% of 1 RPM, 4 } \\
\text { times In 20-30 sec, 12rep/set, } \\
\text { 2times/week for 6week }\end{array}$ \\
\hline & & C: control & 15 & $\begin{array}{c}\text { only counseling and educations, } \\
\text { for 15 mln/once/week }\end{array}$ \\
\hline
\end{tabular}

\section{Data Extraction and Management}

Data was extracted and quality was assessed in accordance with inclusion and exclusion criteria considering standardized protocols. A standardized assessment form was constructed in order to maintain validity and accuracy of data, extracted from the research articles. Furthermore, information was included as the name of the first author, year of publication, targeted population, sample size and its distribution in groups; and type of exercise regime as intervention as represented in table 1.

\section{RESULTS}

\section{Selection of studies included}

Originally, 46 research articles were extracted though an electronic search on various databases like Cochrane, Pubmed, Google Scholar, Science Direct, Biomed-Central (BMC), Pubmed-Europe, Medline, EMBASE and PEdro. All of these researches were published between 2013 and 2018. However, 13 studies were selected including 2 quasi-experimental and 11 randomized controlled trials after screening. Data was extracted and quality assessment was conducted in accordance with the standardized protocols. Meta-analysis and publication bias evaluation could not be performed due heterogeneity in outcome measures of the studies included in the systemic review.

\section{Synthesis of Results}

Outcome measures were assessed for each study along with the interventions applied to each study group in order to investigate the impact of resistance exercise training on respiratory parameters. Out of total 13 researches, included in the systematic review, 4 studies demonstrated significant impact of resistance training on respiratory parameters such as FEVI, FVC, FEV I/FVC ratio, POuptake, TLCO and MVV. However, the remaining 9 researches revealed non-significant outcomes for the intervention. Furthermore, among the 4 studies showing significant result, 2 of them indicated significant positive changes in POuptake, TLCO and VO2 only, while insignificant for FEV1, FVC, FEV1/FVC ratio. The remaining 2 studies supported the efficacy of resistance training in improving spirometric parameters, specifically FEV1, FVC and FEV1/FVC ratio, as illustrated in table 2.

\section{Risk of Bias and overall study quality}

The risk of Bias and study quality was assessed according to standard checklist as represented in table 3 and diagrammatically in figure 2

\section{Selection Bias \\ Random Sequence Generation}

Random sequence generation for 11 studies (Tartibian, 2018, Moradians V, 2016, Molassiotis A, 2015, Zambom-Farraresi, 2015, Edvardsen, 2014, Rovedder PME, 2014, Sanatana-Sosa, 2013, Maryam, 2013, Vonbank K, 2012 and Aweto AH, 2015) demonstrated low risk of bias. However, 2 studies (Ganesh BR, 2018 and Chun SP, 2015) showed unknown and only one (Pothriat $C, 2015$ ) indicated a high risk of bias.

\section{Allocation concealment}

Allocation Concealment for 11 studies (Tartibian, 2018, Moradians V, 2016, Molassiotis A, 2015, Zambom-Farraresi, 2015, Edvardsen, 2014, Rovedder PME, 2014, Sanatana-Sosa, 2013, Maryam, 2013, Vonbank K, 2012 and Aweto $A H, 2015)$ demonstrated low risk of bias. However, 2 studies (Ganesh BR, 2018 and Chun SP, 2015) showed unknown and only one (Pothriat C, 2015) indicated a high risk of bias.

\section{Performance Bias}

\section{Blinding of participants and personnel}

Blinding of participant and personnel was performed by 4 studies (Tartibian, 2018, Zambom-Farraresi, 2015, Edvardsen, 2014, Rovedder PME, 2014) and demonstrated low risk of bias. Conversely, 7 studies (Ganesh BR, 2018, Moradians V, 2016, Chun SP, 2015, Pothriat C,2015, Maryam, 2013, Vonbank K, 2012, and Aweto HA, 2015) showed unknown and 2 studies (Molassiotis A, 2015 and Sanatana-Sosa, 2013) indicated a high risk of bias. 


\begin{tabular}{|c|c|c|c|c|c|}
\hline S.no & Author & Group & $\mathrm{n}$ & Intervention & Outcomes \\
\hline 1 & Ganesh BR & Experimental & 43 & $\begin{array}{l}10 \text { Lbs sandbag breathing exercise } \\
\text { for } 5-10 \text { minutes, thrice a week for } 4 \\
\text { weeks }\end{array}$ & $\begin{array}{l}\text { FEV1, } \\
\text { FVC,FEV1/FVC } \\
\text { and lumbar } \\
\text { stability } \\
\text { improved }\end{array}$ \\
\hline \multirow[t]{2}{*}{2} & \multirow[t]{2}{*}{$\begin{array}{l}\text { Molassioti } \\
\text { s A }\end{array}$} & intevention & 18 & $\begin{array}{l}\text { IMT sessions weekly for } 12 \text { weeks for } \\
\text { a total of } 30 \mathrm{mins} / \text { day }\end{array}$ & $\begin{array}{l}\text { No obvious } \\
\text { physiological } \\
\text { changes in their } \\
\text { FEV1 or FVC } \\
\text { levels. }\end{array}$ \\
\hline & & control & 18 & & \\
\hline 3 & $\begin{array}{l}\text { Edvardsen } \\
\text { E }\end{array}$ & training & 30 & $\begin{array}{l}\text { high-intensity endurance and } \\
\text { strength training ( } 60 \mathrm{~min} \text {, three } \\
\text { times a week, } 20 \text { weeks) }\end{array}$ & $\begin{array}{l}\text { significant } \\
\text { results for PO } \\
\text { Uptake and , } \\
\text { Tlco, non- } \\
\text { significant for } \\
\text { FEV1, MVV }\end{array}$ \\
\hline 4 & Pothirat C & Experimental & 30 & $\begin{array}{l}\text { incremental strength and endurance } \\
\text { with two } 35-40 \text {-minute sessions per } \\
\text { week for } 8 \text { weeks }\end{array}$ & $\begin{array}{l}\text { No significant } \\
\text { changes in lung } \\
\text { function }\end{array}$ \\
\hline \multirow[t]{2}{*}{5} & $\begin{array}{l}\text { Santana- } \\
\text { Sosa E }\end{array}$ & training & 10 & $\begin{array}{l}\text { Aerobic }+ \text { strength training } 3 \\
\text { day/week }\end{array}$ & \multirow{2}{*}{$\begin{array}{l}\text { Significant } \\
\text { results in PImax } \\
\text { \&VO2 max and } \\
\text { non-significant } \\
\text { in FEV1 and FVC }\end{array}$} \\
\hline & & control & 10 & No intervention & \\
\hline \multirow[t]{2}{*}{6} & $\begin{array}{l}\text { Zambom- } \\
\text { Ferraresi F }\end{array}$ & $\begin{array}{l}\text { combined } \\
\text { training }\end{array}$ & 14 & $\begin{array}{l}12 \text { weeks, I d / week resistance } \& 1 d \text { / } \\
\text { week endurance training }\end{array}$ & \multirow{2}{*}{ non- significant } \\
\hline & & $\begin{array}{l}\text { resistance } \\
\text { training }\end{array}$ & 14 & $\begin{array}{l}12 \text { weeks, } 2 \text { d / week resistance } \\
\text { training }\end{array}$ & \\
\hline \multirow[t]{2}{*}{7} & $\begin{array}{l}\text { Rovedder } \\
\text { PME }\end{array}$ & exercise & 19 & $\begin{array}{l}\text { home based exercise; a combination } \\
\text { of aerobic and strength training }\end{array}$ & $\begin{array}{l}\text { non-significant } \\
\text { FEV1, FVC }\end{array}$ \\
\hline & & control & 22 & normal routine practices & \\
\hline \multirow[t]{3}{*}{8} & $\begin{array}{l}\text { Moradians } \\
\text { V }\end{array}$ & aerobic & 12 & $\begin{array}{l}\text { aerobic ex } 40-60 \min 3 / \text { week (10 } \\
\text { min WU, } 20 \text { min AA @70-80\% MHR }\end{array}$ & \multirow{3}{*}{$\begin{array}{l}\text { non-significant } \\
\text { value for } \\
\text { spirometric } \\
\text { values }\end{array}$} \\
\hline & & resistance & 12 & $\begin{array}{l}\text { weight training } 3 \text { sets } 10 \text { reps of each } \\
\text { ex, intensity increased by }\end{array}$ & \\
\hline & & interval & 12 & $\begin{array}{l}\text { Short periods of running at } 85 \% \\
\text { maximum heart rate. Each session } \\
\text { started with warm up, which was } \\
\text { followed by five rounds of running } \\
20,30,60 \text {, and } 100 \text { meters, and } \\
\text { finished with cool down exercises. }\end{array}$ & \\
\hline
\end{tabular}




\begin{tabular}{|c|c|c|c|c|c|}
\hline 9 & Chun SP & experimental & 15 & $\begin{array}{l}\text { Core stability exercises } 30 \text { min / } \\
\text { session, } 4 \text { session / week for } 8 \text { weeks }\end{array}$ & \multirow{2}{*}{$\begin{array}{l}\text { Significant } \\
\text { increase in } \\
\text { Pulmonary } \\
\text { Function }\end{array}$} \\
\hline & & control & 15 & general exercises & \\
\hline \multirow[t]{3}{*}{10} & Vonbank K & $\begin{array}{l}\text { endurance } \\
\text { training }\end{array}$ & 12 & $\begin{array}{l}\text { cycle ergometer twice / week total } \\
\text { duration } 60 \text { min intensity } 60 \%\end{array}$ & \multirow{3}{*}{ Non-significant } \\
\hline & & $\begin{array}{l}\text { progressive } \\
\text { strength } \\
\text { training }\end{array}$ & 12 & twice/ week 8-15 rep / set & \\
\hline & & Combination & 12 & both combined & \\
\hline \multirow[t]{3}{*}{11} & Tartibian & HIIT & 12 & $\begin{array}{l}30 \mathrm{~min} / \text { day, } 3 \text { days/week at } 60-90 \% \text { of } \\
\text { heart rate reserve for } 8 \text { weeks }\end{array}$ & \multirow{3}{*}{ significant } \\
\hline & & RT & 12 & $\begin{array}{l}60 \mathrm{~min} / \text { day, } 3 \text { days/week by } 60 \%-90 \% \\
\text { of } 1 \mathrm{RM} \text { for } 8 \text { weeks }\end{array}$ & \\
\hline & & NON-EX & 12 & No intervention & \\
\hline \multirow[t]{4}{*}{12} & Khosravi M & Control & 9 & no intervention & \\
\hline & & $\begin{array}{l}\text { ET (exercise } \\
\text { training) }\end{array}$ & 9 & $\begin{array}{l}\text { ( } 8 \text { weeks, } 3 \text { sessions/week) for ET was } \\
20-26 \text { min/session running with 60- } \\
80 \% \text { maximum heart rate (HR max }\end{array}$ & \multirow{3}{*}{$\begin{array}{l}\text { non-Significant } \\
\text { for FEV1/FVC }\end{array}$} \\
\hline & & $\begin{array}{l}\text { RT } \\
\text { (resistance } \\
\text { training) }\end{array}$ & 10 & $\begin{array}{l}\text { two circuits/session, } 40-60 \text { s for each } \\
\text { exercise with } 60-80 \% \text { one repetition } \\
\text { maximum (1RM), } 1 \text { and } 3 \text { minutes } \\
\text { active rest between exercises and } \\
\text { circuits respectively }\end{array}$ & \\
\hline & & ERT & 9 & $\begin{array}{l}\text { in agreement with either ET or RT } \\
\text { protocols, but the times of running and } \\
\text { circuits were half of ET and RT }\end{array}$ & \\
\hline \multirow[t]{3}{*}{13} & Aweto HA & A: aerobic & 15 & $\begin{array}{l}\text { Aerobic exercise; treadmill, } 10 \mathrm{~min} \text { for } \\
4 \text { week, } 15 \mathrm{~min} \text { for } 2 \text { week, twice a for } 6 \\
\text { week total }\end{array}$ & \multirow{3}{*}{ non-significant } \\
\hline & & B: resistance & 15 & $\begin{array}{l}\text { Dumbbell, } 50-70 \% \text { of } 1 \text { RPM, } 4 \text { times in } \\
20-30 \mathrm{sec}, 12 \mathrm{rep} / \mathrm{set}, 2 \text { times/week for } \\
6 \text { week }\end{array}$ & \\
\hline & & C: control & 15 & $\begin{array}{l}\text { only counseling and educations, for } 15 \\
\text { min/once/week }\end{array}$ & \\
\hline
\end{tabular}




\begin{tabular}{|c|c|c|c|c|c|c|}
\hline Domains & $\begin{array}{l}\text { Rando } \\
\text { m } \\
\text { Sequen } \\
\text { ce } \\
\text { Genera } \\
\text { tion }\end{array}$ & $\begin{array}{l}\text { Allocation } \\
\text { Concealm } \\
\text { ent }\end{array}$ & $\begin{array}{l}\text { Blinding of } \\
\text { Participants } \\
\text { and } \\
\text { Personnel }\end{array}$ & $\begin{array}{l}\text { Blinding } \\
\text { of } \\
\text { Outcome } \\
\text { Assessm } \\
\text { ent }\end{array}$ & $\begin{array}{l}\text { Incompl } \\
\text { ete } \\
\text { Outcome } \\
\text { Data }\end{array}$ & $\begin{array}{l}\text { Selective } \\
\text { Reportin } \\
\text { g }\end{array}$ \\
\hline Ganesh BR, 2018 & $?$ & $?$ & $?$ & ? & $\boldsymbol{V}$ & $\checkmark$ \\
\hline Tartibian, 2018 & $\boldsymbol{V}$ & $\boldsymbol{V}$ & $\checkmark$ & $\checkmark$ & $\boldsymbol{V}$ & $\checkmark$ \\
\hline $\begin{array}{l}\text { Moradians V, } \\
2016\end{array}$ & $\checkmark$ & $\checkmark$ & $?$ & $?$ & $\boldsymbol{v}$ & $\boldsymbol{V}$ \\
\hline Chun SP, 2015 & $?$ & $?$ & $?$ & $?$ & $\checkmark$ & $\checkmark$ \\
\hline $\begin{array}{l}\text { Molassiotis A, } \\
2015\end{array}$ & $\checkmark$ & $\boldsymbol{v}$ & $\mathrm{x}$ & $x$ & $\checkmark$ & $\checkmark$ \\
\hline Pothirat C, 2015 & $x$ & $x$ & $?$ & $?$ & $\boldsymbol{v}$ & $\checkmark$ \\
\hline $\begin{array}{l}\text { Zambom- } \\
\text { Ferraresi, } 2015\end{array}$ & $v$ & $v$ & $\checkmark$ & $\checkmark$ & $\boldsymbol{v}$ & $\boldsymbol{V}$ \\
\hline Edvardsen, 2014 & $\boldsymbol{V}$ & $\boldsymbol{v}$ & $\checkmark$ & $\mathrm{x}$ & $\checkmark$ & $\checkmark$ \\
\hline $\begin{array}{l}\text { Rvendder PME, } \\
2014\end{array}$ & $v$ & $v$ & $\checkmark$ & $\checkmark$ & $\boldsymbol{v}$ & $\checkmark$ \\
\hline $\begin{array}{l}\text { Sanatana-Sosa, } \\
2013\end{array}$ & $\boldsymbol{v}$ & $\checkmark$ & $x$ & $\checkmark$ & $\boldsymbol{v}$ & $\checkmark$ \\
\hline Maryam, 2013 & $\boldsymbol{V}$ & $\boldsymbol{V}$ & $?$ & $?$ & $\boldsymbol{V}$ & $\checkmark$ \\
\hline Vonbank K, 2012 & $\checkmark$ & $\checkmark$ & $?$ & $?$ & $\checkmark$ & $\checkmark$ \\
\hline Aweto HA, 2015 & $\boldsymbol{V}$ & 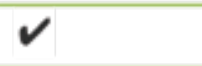 & $?$ & $?$ & $\checkmark$ & $\checkmark$ \\
\hline
\end{tabular}

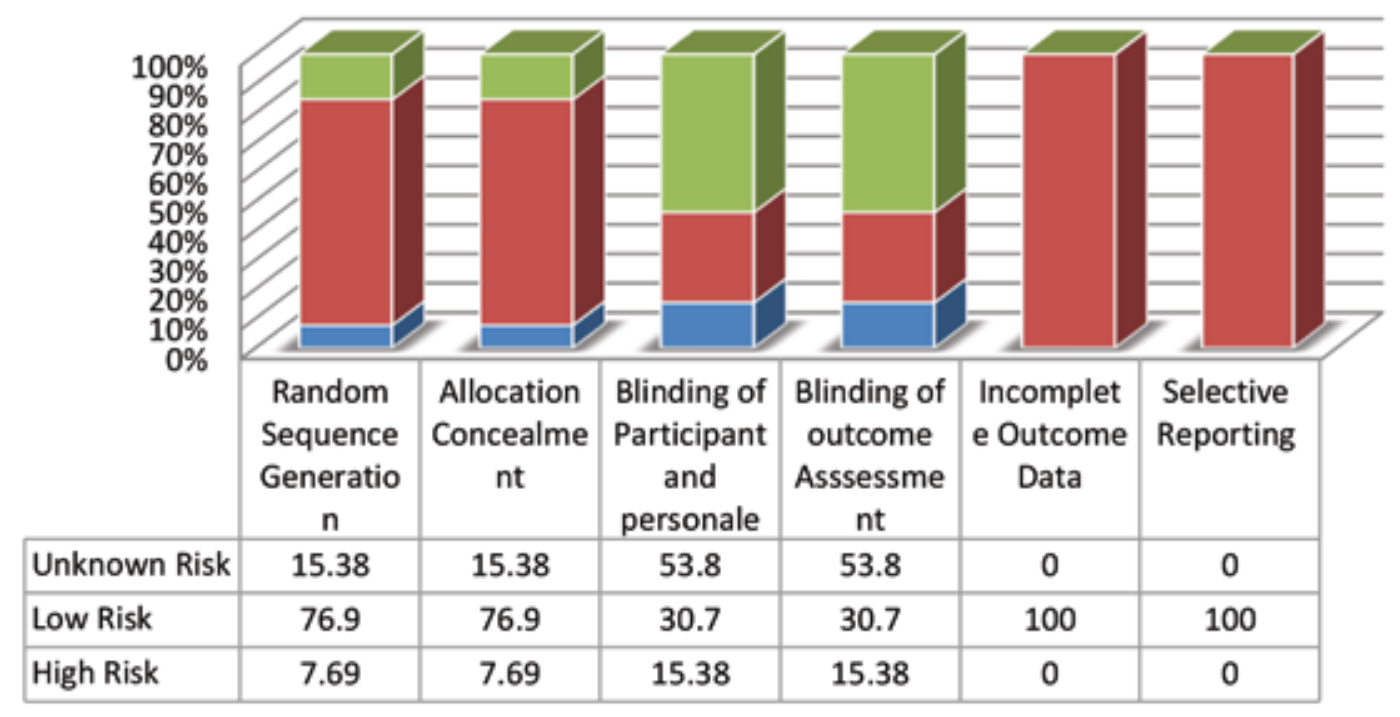

Figure2: Authors perception for risk of bias 


\section{Detection Bias}

\section{Blinding of Outcome Assessment}

Blinding of outcome Assessment was performed by 4 studies (Tartibian, 2018, Zambom-Farraresi, 2015, Sanatana-Sosa, 2013, performed by 4 studies (Tartibian, 2018, Zambom-Farraresi, 2015, Sanatana-Sosa,' 2013, Rovedder PME, 2014) and demonstrated low risk of bias. Conversely, 7 studies (Ganesh BR, 2018, Moradians V, 2016, Chun SP, 2015, Pothriat C, 2015, Maryam, 2013, Vonbank K, 2012, and Aweto HA, 2015) showed unknown and 2 studies (Molassiotis A, 2015 and Edvardsen, 2014) exhibited a high risk of bias.

\section{Attrition Bias \\ Incomplete Outcome Data}

None of the studies presented incomplete outcome data. All 13 studies (Tartibian, 2018, Zambom-Farraresi, 2015, Sanatana-Sosa, 2013, Rovedder PME, 2014, Ganesh BR, 2018, Moradians V, 2016, Chun SP, 2015, Pothriat C, 2015, Maryam, 2013, Vonbank K, 2012, and Aweto HA, 2015, Molassiotis A, 2015 and Edvardsen, 2014) indicated low risk of bias.

\section{Reporting Bias \\ Selective Reporting}

Selective reporting for all 13 studies (Tartibian, 2018, Zambom-Farraresi, 2015, Sanatana-Sosa, 2013, Rovedder PME, 2014, Ganesh BR, 2018, Moradians V, 2016, Chun SP, 2015, Pothriat C, 2015, Maryam, 2013, Vonbank K, 2012, and Aweto HA, 2015, Molassiotis A, 2015 and Edvardsen, 2014) indicated low risk of bias.

\section{DISCUSSION}

This systematic review has explored the impact of anaerobic resistance training exercise regime on various respiratory parameters including Forced Vital Capacity (FVC), Forced Expiration volume in first second (FEV1), Vital Capacity (VC), Minute ventilation (MV), Maximal Voluntary Ventilation (MVV), Maximum Oxygen consumption (VO2max), Maximum Pulmonary Inspiration (PImax), Maximum Pulmonary Expiration (PEmax) and Transfer factor for carbon monoxide (TLCO). However, meta-analysis could not be performed due to the heterogeneity of the outcome measures, interventions and targeted populations of the studies included in this systematic review.

The convergence of the findings of this systematic review revealed a low evidence of resistive exercise training regimes having a profound impact in improving respiratory parameters. Moreover, the studies demonstrating significant impact of resistance training on pulmonary system, also included those in which resistive training and aerobic were used in combination. Probably, the heterogeneous nature of target population of different researches included in the systematic review has stipulated the differences in result outcomes, since different pulmonary parameters respond distinctly to the same intervention.

Interestingly, a recently conducted meta-analysis, concluded with the effectiveness of exercise training (i.e. the combination of aerobic and anaerobic/ resistance exercise training) in improving the pulmonary function of individuals suffered with chronic obstructive pulmonary disease (COPD) ${ }^{35}$ In addition to this the study also deduced that exercise training has more profound effect alone than in combination with other regimes of treatment interventions ${ }^{35}$. The distinctive feature of this study was the systematic characterization of outcome measures that might be the reason of clear results.

Another systematic review and meta-analysis has deduced more specifically the positive impact of resistance exercise training in COPD patients. The review postulated results inquiring 7 outcomes including 2 primary and 5 secondary outcome measures. The prominent significant enhancement was demonstrated in FEV , with $\mathrm{p}$-value of 0.04 and annulled the possibility of adversity in including resistance training in pulmonary rehabilitation regime ${ }^{36}$.

Another challenging concept has been discussed in a research study that if there is a significant difference in the effects of high and low intensity upper and lower limb extension exercise considering various cardiovascular outcome measures like minute ventilation (MV), Oxygen Uptake (VO2 peak) and heart rate (HR). Subsequently, it revealed the absence of any relationship between intensity of exercise and acute cardiorespiratory changes in COPD patients. However, it does not discussed long-term effects for this intervention ${ }^{37}$. Meanwhile another study studied the impact of number and position of limbs engaged in the short bout resistive exercise on similar pulmonary parameters including $\mathrm{FEV} 1, \mathrm{MV}, \mathrm{VO}_{2 \text { peak }}$ and saturation of oxygen and concluded no significant impact of number of limbs engaged in exercise ${ }^{38}$.

\section{CONCLUSION}

Finally, the findings of systematic review conducted to investigate the effects of resistive exercise alone or in combination with aerobic regimes on respiratory parameters, concluded that there is a low evidence for the impact of resistance exercises in improving lung function and respiratory parameters. However, the indication of significant results of resistive exercises in improving lung function is a suggestive of future need of further researches to be conducted on this subject. 


\section{REFERENCES}

[1] Hillsdon MM, Brunner EJ, Guralnik JM, Marmo† MG. Prospective study of physical activity and physical function in early old age. Am J of Prev Med. 2005;28(3):245-50.

[2] Badaam Khaled M, Munibuddin A, Khan ST, Choudhari SP, Doiphode R. Effect of traditional aerobic exercises versus sprint interval training on pulmonary function tests in young sedentary males: a randomized controlled trial. JCDR. 2013;7(9):1890.

[3] Doijad VP, Kamble P, Surdi AD. Effect of Yogic Exercises on Aerobic Capacity (VO^ sub $2 \wedge$ max). International Journal of Physiology. 2013;1 (2):47.

[4] Saravanan S, Umesh T M, Rakesh. Aerobic training for health and fitness. J Arts Cult.2011; 2(1-3):65.

[5] American College of Sports Medicine. Guidelines for exercise testing and prescription. 3rd ed. Philadelphia: Lea and Febiger, 1988:168-169

[6] Guyton and Hall. Textbook of medical physiology. Eleventh edition Noida: Published by Elsevier; 2008:1061

[7] Park J, Han D. Effects of high intensity aerobic exercise on treadmill on maximum-expiratory lung capacity of elderly women. J PHYS THER SCl. 2017;29(8):1454-7.

[8] Khashaba AS. Effect of levels of physical activity on pulmonary function of male Saudi university students. J Sports Sci. 2015;5(5):209-12.

[9] Azad A, Gharakhanlou R, Niknam A, Ghanbari A. Effects of aerobic exercise on lung function in overweight and obese students. Journal of respiratory disease, thoracic surgery, intensive care, tuberculosis. $2011 ; 10(3): 24-31$.

[10] Mottram C. Ruppel's Manual of Pulmonary Function Testing 10: Ruppel's Manual of Pulmonary Function Testing. Elsevier Health Sciences; 2013.

[11] Gloeckl R, Marinov B, Pitta F. Practical recommendations for exercise training in patients with COPD. Eur Respir Rev. 2013;22(128):179.

[12] Nici L, Donner C, Wouters E, Zuwallack R, Ambrosino N, Bourbeau J, Carone M, Celli B, Engelen M, Fahy B, Garvey C. American thoracic society/European respiratory society statement on pulmonary rehabilitation. Am J Respir Crit Care Med. 2006;173(12):1390-413.

[13] Mazic S, Lazovic B, Djelic M, Suzic-Lazic J, Djordjevic-Saranovic S, Dumric T. Respiratory parameters in elite athletes - does sport have an influence? Revista Portuguesa de Pneumologia (English Edition). 2015;21 (4):192-197.

[14] Langer D, Hendriks EJ, Burtin C, Probst V, Van der Schans CP, Paterson WJ, Verhoef-de Wijk MC, Straver RV, Klaassen M, Troosters T, Decramer M. A clinical practice guideline for physiotherapists treating patients with chronic obstructive pulmonary disease based on a systematic review of available evidence. Clin Rehabil. 2009;23(5):445-62.

[15] Larson JL, Kim MJ, Sharp JT, Larson DA. Inspiratory muscle training with a pressure threshold breathing device in patients with chronic obstructive pulmonary disease. Am Rev Respir Dis. 1988;138(3):689-96.

[16] Kim E, Lee $\mathrm{H}$. The effects of deep abdominal muscle strengthening exercises on respiratory function and lumbar stability. J Phys Ther Sci. 2013;25(6):663-5.

[17] O'Shea SD, Taylor NF, Paratz JD. Progressive resistance exercise improves muscle strength and may improve elements of performance of daily activities for people with COPD: a systematic review. Chest. 2009; 136(5):1269-83.

[18] Probst VS, Troosters T, Pitta F, Decramer M, Gosselink R. Cardiopulmonary stress during exercise training in patients with COPD. Eur. Respir. J. 2006; 27(6):1110-1118

[19] Tartibian B, Khayat SM. Respiratory Indexes and Inflammatory Marker Change by Different Exercise Training. Iran J Allergy Asthma Immunol. 2018; $17(2): 227$.

[20] Bittencourt HS, Cruz CG, David BC, Rodrigues-Jr E, Abade CM, Junior RA, Carvalho VO, dos Reis FB, Gomes Neto M. Addition of non-invasive ventilatory support to combine aerobic and resistance training improves dyspnea and quality of life in heart failure patients: a randomized controlled trial. Clin Rehabil. 2017:31(11):1508-15

[21] Küçükçakır N, Altan L, Korkmaz N. Effects of Pilates exercises on pain, functional status and quality of life in women with postmenopausal osteoporosis. J Bodyw Mov Ther. 2013;17(2):204-1 1

[22] Ganesh BR, Gada Y, Dhargalkar S, Rajpurohit D. Effect Of Sandbag Breathing Exercise On Respiratory Parameters And Lumbar Stability In Asymptomatic Individuals-An Experimental Study. Int. J. Curr. Res. Med. Sci. 2018; 4(2):75-80. Molassiotis A, Charalambous A, Taylor P, Stamataki Z, Summers Y. The effect of resistance inspiratory muscle training in the management of breathlessness in patients with thoracic malignancies: a feasibility randomised trial. Support Care Cancer. 2015;23(6):1637-45.

[24] Edvarsen E, Skjonsberg OH, Holme I, Nordsletten L, Borchenius F, Anderssen SA. High intensity training following lung cancersurgery: a randomized controlled trial. Thorax. 2014;0: 1-7.

[25] Pothirat C, Chaiwong W, Phetsuk N. Efficacy of a simple and inexpensive exercise training program for advanced chronic obstructive pulmonary disease patients in community hospitals. J Thorac Dis. 2015;7(4):637.

[26] Santana-Sosa E, Gonzalez-Saiz L, Groeneveld IF, Villa-Asensi JR, de Aguero Barrio MG, Fleck SJ, López-Mojares LM, Pérez M, Lucia A. Benefits of combining inspiratory muscle with whole 
muscle training in children with cystic fibrosis: a randomised controlled trial. $\mathrm{Br} \mathrm{J}$ sports Med. 2014;48(20):1513-1517.

[27] Zambom-Ferraresi F, Cebollero P, Gorostiaga EM, Hernández M, Hueto J, Cascante J, Rezusta L, Val L, Anton MM. Effects of Combined Resistance and Endurance Training Versus Resistance Training Alone on Strength, Exercise Capacity, and Quality of Life in Patients With COPD. . J Cardiopulm Rehabil Prev. 2015;35(6):446.

[28] Rovedder PM, Flores J, Ziegler B, Casarotto F, Jaques P, Barreto SS, Dalcin PD. Exercise programme in patients with cystic fibrosis: a randomized controlled trial. Respir Med. 2014;108(8):1134-40.

[29] Moradians V, Rahimi A, Moosavi SA. Effect of eight-week aerobic, resistive, and interval exercise routines on respiratory parameters in non-athlete women. Tanaffos. 2016;15(2):96.

[30] Kim KY, Chun SP, Kang TG, Kim GD.Effects of Core Stability Training on Postural Control Ability and Respiratory Function in Chronic Stroke Patients. Healthcare and Nursing. 2015;88(1):181-186

[31] Vonbank K, Strasser B, Mondrzyk J, Marzluf BA, Richter B, Losch S, Nell H, Petkov V, Haber P. Strength training increases maximum working capacity in patients with COPD-randomized clinical trial comparing three training modalities. Respir Med. 2012;106(4):557-63.

[32] Tartibian B, Khayat SMA. High-Intensity Interval Training / Resistance Exercise Lead to Greater Lung Function: Improvement of FEVI/ FVC\% and FEF25-75\%. Iran J Allergy Asthma Immunol. 2018;17(1):224-224Khosravi M, Tayebi SM, Safari

[33] H. Single and concurrent effects of endurance and resistance training on pulmonary function. Iran J Basic Med Sci. 2013;16(4):628

[34] Aweto HA, Akodu AK, Adedara AC, Olawale OA. A comparative study of the effects of aerobic and resisted exercises on selected cardiopulmonary parameters in patients with asthma. African Journal of Physiotherapy and Rehabilitation Sciences. 2015;7(1-2):52-60.

[35] Salcedo PA, Lindheimer JB, Klein-Adams JC, Sotolongo AM, Falvo MJ. Effects of Exercise Training on Pulmonary Function in Adults with Chronic Lung Disease: a meta-analysis of Randomizes Controlled Trails. Arch Phys Med Rehabil.2018;99(12):2561-2569

[36] Liao W, Chen J, Chen X, Lin L, Yan H, Zhou Y et.al. Impact of Resistance training in subjects with COPD: A Systematic Review and Meta-Analysis. Respcare.2015;60(8):1 130-1 145

[37] Robles P, Araujo T, Brooks D, Zabjek K, Janaudis-Ferrwira T, Marzolini S. et.al. Cardiorespiratory Responses to Short Bouts of Resistance Training Exercises in Individuals with Chronic Obstructive Pulmonary Disease; a Comparison of Exercise Intensities. J Cardiopulm Rehabil Prev. 2017;37(5):356-362.

[38] Robles P, Araujo T, Brooks D, Zabjek K, Janaudis-Ferreira T, Marzolini S, et.al. Does limb partitioning and positioning affect acute cardiorespiratory responses during strength exercises in patients with COPD? Respiro RESPIROLOGY. 2017;22(7):1336-1342. 